\title{
Mental Health Recovery in SDGs Through the Empowering Family Communication in Palmerah Community Jakarta
}

\author{
Z. Hidayat ${ }^{1 *}$, Triasesiarta Nur ${ }^{2}$, Fransiska A. Kusumastuti $^{3}$, Debra Hidayat ${ }^{4}$ \\ \{z.hidayat@binus.edu $\left.{ }^{1}\right\}$ \\ Senior Lecturer at Communication Department, BINUS Graduate Program-Master of Strategic \\ Marketing Communication, Bina Nusantara University, Jakarta Indonesia $11480^{1}$ \\ Senior Lecturer at Accounting Finance Department, Faculty of Economic and Communication, Bina \\ Nusantara University, Jakarta Indonesia $11480^{2}$ \\ Lecturer at Elementary School Teacher Education Department, Faculty of Humanity, Bina Nusantara \\ University, Jakarta Indonesia $11480^{3}$ \\ Student at Communication Department, BINUS Graduate Program-Master of Strategic Marketing \\ Communication, Bina Nusantara University, Jakarta Indonesia $11480^{4}$
}

\begin{abstract}
This study analyzes the mental health recoveries among adolescents, such as addiction to internet consumption through social media, websites, games, YouTube, Netflix, and live streaming. Action research was carried out on communities in two villages involving 22 families as participants, and each family has one to four children. Ethnography was also used to collect data from observations, interviews, and researchers who are active in the community for three months. The first step is to identify the problem and then carry out an extension and mentoring program. Researchers applied a program to improve interaction and communication patterns in families, both dyadic and group. The empowering families and community are conducted through day-to-day coaching to the adolescents to improve communication patterns and relationships between family members, increasing knowledge about communication technology's function, and strengthening cultural ties face-to-face and online. The results showed that mental health recovery loosened dependence on online games and the duration of social media chats. The community shows the improvement of interaction and relationship patterns in family communication. Meanwhile, dimensions such as knowledge about the role of communication technology and cultural ties in the community are considered necessary by the community to improve adolescents' mental health.
\end{abstract}

Keywords: Adolescence, Internet addiction, Family communication, Mental health, Hyperactivity online

\section{Introduction}

Sustainable development goals (SDGs) are becoming increasingly important to the attention of all stakeholders. The government, society, universities, and non-government organizations (NGOs) are responsible for preserving nature. Because economic development with the exploitation of nature must pay attention to balance and avoid damage to ecosystems, on a macro scale, attention to the ecosystem's balance will result in the harmony of life between 
humans and their environment. Human welfare can be seen from its ability to develop health, education, poverty reduction, natural resource utilization, and eco-friendly behavior.

On a micro level, every family faces a mental health issue, even though its role is essential to building family welfare and happiness. Each family's welfare reflects the success of the SDGs on a micro-scale by paying attention to individuals' daily lives, such as both parents and children in a nuclear family. Attention to the micro-level is significant because it can show considerable success for the SDGs, which was then seen at the community level in an area.

Initial observations were made to collect data in identifying problems faced by families and communities in Palmerah County, West Jakarta. This area has demographic characteristics that are relatively the same as other areas in Metropolitan Jakarta. However, its peculiarity is that it is a community close to universities, traditional markets, modern markets, and close to offices centers. Researchers chose these characteristics to understand the problems at hand, from the perspective of SDGs and at the same time providing a capacity-building program as a series of action research.

The topic of mental health in family and community came into sight at the initial review on the field, from the perspective of Sustainable Development Goals (SDGs) as a United Nations Agreement through planning, implementation, control, evaluation, and improvement in all countries. With this viewpoint, attention was paid to preserving the blue planet, where humans benefit but must balance environmental preservation. SDGs, as we know, on the macro-side efforts, are made to improve with poverty reduction, good marine resource management, the government pro-poor strategies and policies, food security, and nutrition. Moreover, on the micro-side, we understand that every family has an essential role in achieving welfare and happiness, which is in line with mental health.

Regarding mental health, we discuss this issue from the study of communication disciplines, namely media consumption by adolescents in the family. The age of children, teenagers, and young adults were critical for forming a human character, so this study is essential to discuss mental health. The internet's use is massive and of long duration in everyday life, including online games, YouTube, Instagram, Line, WA, Twitter, etc. This research article focuses on an action research approach through a capacity building program.

From the perspective of the communication discipline, mental health views a lack of theory and people habits related to online media such as being addicted to online games, enduring long hours of social media chatting, live streaming on YouTube, and other applications. All these online activities dominate family members, both father, mother, and children, including adolescents who have to divide their time to learn and play offline for their mental health.

Various previous studies have discussed mental health from a health and medical perspective without analyzing media use by adolescents in their families. Simultaneously, physical and mental health factors are related to family members' discipline in daily life. The communication science perspective highlights the impact of online media's excessive use on making children and adolescents undisciplined. There was a lack of communication interactions with their parents as a contributing factor to mental unhealthiness.

The previous research on depression by Dong-Woo et al. does not prioritize the importance of family communication and several other studies. By directing children and guiding them to get used to a balanced communication interaction with parents and peer groups, mental health recovery can be achieved without neglecting media technology's role in the learning process and community development. The still rare attention to the importance of improving family communication that balances online and offline life is discussed in this research article making this topic have novelty and distinctiveness. 
This research article thoroughly highlights the question: How is the role of empowering family communication for mental health recovery as the result of excessive use of online media and communication interactions that do not consider the role of parental assistance? Therefore, this research article aims to analyze the mental health recovery among adolescents with capacity building treatment and sharing knowledge about the dangers of mental health problems such as depression when consuming the internet without a break. Addiction to online media in the family includes internet consumption through social media, websites, games, YouTube, Netflix, and live streaming.

\section{Literature review}

\subsection{Computer-mediated communication and internet addiction}

Sheizaf Rafaeli, in Holmes [12], was referred to as the prominent theorist who explains computer-mediated communication (CMC) since 1988, which distinguishes between connectivity, reactivity, and interactivity. In his opinion, the network must have a human interface, but it must also have an architecture that allows interactivity. Interactive networks form a relationship that is strengthened by interactive two-way communication. However, two way communication does not in itself guarantee interactivity. Instead, exchange or action reaction should develop into a relationship where one speech becomes the context for another. Without this form of connectivity, relationships are circular or solipsistic.

Furthermore, Holmes [12] explains that online interactivity is distributed across the network and cannot be reduced to the number of point-to-point exchanges. Each message takes into account the previous message and the way the previous messages react to one another. This research article adopts online communication or CMC in addition to new media theory as a basis for discussing interactivity, expression, synchronicity, realtime, and so on. Gen Z understood the expression and interactivity of social learning and its effect on the environment's advocacy movement in terms of CMC and the new media theories.

The concept of "internet addiction" is still an exciting discussion and continues to be questioned. However, depressed or isolated individuals may be more likely to focus on specific activities excessively. Bell [2], for example, revealed that there are extreme communities that use online media, so that internet therapy is needed to maintain mental health. Several "extreme communities" have also formed online, such as pro-anorexia, pro-suicide, proamputation, and possibility-psychotic groups. Previous research findings like these serve to provide support beyond the medical and social mainstream who find their beliefs and behaviors unacceptable.

The risks faced by children and adolescents can be said to be balanced with the benefits obtained from the internet, especially if school or campus policies move physical classrooms to virtual classrooms in the teaching and learning process. This research article puts forward mental health problems due to internet addiction (IA) or specifically online games addiction, YouTube, chatting, and so on, creates the behavioral problem, particularly among adolescents. Meanwhile, the word "addiction" carries a negative stigma as a compulsive behavior towards a family's life.

While the use of technology as a learning tool holds great promise for children, misuse of technology can have the opposite effect. Young and De Abreu [38] show that too much children's time was spent in front of gadget screens, which results in a lack of school performance such as poor grades, low reading scores, inattention, blunt thinking, and social 
problems. Online gadget exposure is also added to watching television and other offline video games that interfere with healthy eating and sleeping habits for children and adolescents to complete their homework. Several previous studies have also concluded that time spent staring at gadget screens can rob children and teens of opportunities to develop essential learning skills. The assumption is that excessive media use impacts decreasing physical and mental health and that indiscipline also affects communication patterns and relationships in the family.

\subsection{Mental health}

Mental health is a state of well-being in which individuals are aware of their own abilities, can cope with the pressures of normal life, can work productively and be fruitful, and are able to contribute to their communities [34]. Mental health concept is officially used because according to WHO, there is no opposite concept such as "mental illness" in the medical world. However, the definition of this concept is feared to cause misunderstanding so that two aspects of the coverage referred to by WHO are emphasized, namely positive emotions and complementary functions. Three components of mental health [11], [15], [16] are emotional well-being, psychological well-being, and social welfare. Emotional wellbeing includes happiness, interest in life, and satisfaction, while psychological well-being, such as liking a large part of a person's personality, being good at managing daily life responsibilities, having good relationships with others, and feeling satisfied with one's own life. Lastly, well-being social sense refers to positive functions which include having something to contribute to society (social contribution), feeling part of the community (social integration), believing that society is a better place for everyone (social actualization), and that society works, and makes sense to them (social coherence). This article discusses micro mental health within the family in a community associated with excessive media use with a negative impact.

While representing substantial progress concerning moving away from mental health's conceptualization as a state of absence of mental illness, this mental health concept raises several concerns. It lends itself to potential misunderstandings when it identifies positive feelings and positive functioning as critical factors for mental health. Understanding mental health associated with internet addiction reflects the causality that IA affects young people's physical, mental, and social conditions. Online and digital life has grown as the internet culture, so that researchers like Kirmayer [17] highlight the sides of identity and community to discuss mental health.

\subsection{Community}

Tracy [33] describes several definitions of community: 1) A community is a collection of people who inhabit a particular geographic place as a geographical unit larger than family and neighborhood but smaller than the state in local units such as cities, towns, and school districts. 2) As a frame of reference for a group of people who have different cultural identities. 3) As a group of people who share the same interests or activities (for example, the snowboarding community, the Facebook community, the vegan community). 4) As a positive sentiment that can be applied, achieved, pursued, or threatened with extinction. 5) As a pillar in two pairs of interdependent and controversial political values to realize a commitment to a group's welfare (community), it was contrasted with assessing individual rights. The definition of the community, thus for Gen Z, is more as a group of people with the same relative age with interest in paying attention to environmental issues or an eco-friendly lifestyle. It means that the third definition is closer to the discussion of this article. 


\subsection{Capacity building}

The capacity building definition refers to efforts to strengthen community capacity by determining their values and priorities. Capacity building-based development is carried out with separate interventions - such as those developed by Oxfam, an NGO - characterized by its own fundamental beliefs. Capacity building believes that all people have the right to a fair share of the world's resources. People will become writers of development about their story themselves, and that denial of rights is at the heart of poverty and suffering. Even though specific essential capacities (social, economic, political, and practical) upon which development relies, Oxfam strives to support organizations working for sustainable social justice [6].

The SDGs implementation is seen as more appropriate to be approached with the concept of 'capabilities approach' as put forward by Amartya Sen - a philosopher and economist who won the Nobel Prize. His work on 'rights' has been instrumental in analyzing economic inequalities such as hunger and its prevention solutions. According to Sen, the goal of development is to improve the community's quality of life, which was best achieved by providing vulnerable communities access to a broader spectrum of capabilities. Furthermore, the 'capabilities approach' is related to (i) expanding goods and services, (ii) increasing utility, or (iii) meeting basic needs [6]. In community capacity building consisting of families facing mental health, this research article considers recovery and balancing necessary to achieve excellent mental health for adolescents by improving family communication patterns online and offline.

\subsection{Family communication}

From the point of view of communication studies, this research article needs to put forward Family Communication Theory as a process and family relationships that explain phenomena of interests, which frequently are the relationships or communication behaviors themselves or their outcomes for family members. Family communication theory is a communication process within the family and family relationships that explains the phenomenon of interest among family members. Furthermore, family communication is also the relationship or communication behavior itself or the result for family members. Thus, Family Communication Theory is critical in understanding the process of interaction in the family, its results, and benefits that are influenced and generated [18].

The qualitative approach to understanding family communication focuses on human behavior expressed in interactions and ultimately relies on the specific processes of participant interaction in the family's structural control. According to Koerner [18], family communication patterns are associated with several processes for forming the family as a community institution. The process that takes up several challenges such as conflict, affirmation, and strengthening and maintenance of affection was continued with family rituals and understanding. Communication is also associated with relationship outcomes, such as the presence of children. There can also be communication fears, conflicts, romantic partner interactions, resilience, and children's mental and physical health. So, the family communication theory also pays attention to the mental and physical health of family members. In this article, the analysis was deepened by using excessive digital technology in the family.

The family communication pattern is associated with the frequency of adolescent-parent communication directly, via telephone, text message, and email. Rudi et al. [28] analyzed adolescent-parent communication in a digital world by linking family communication patterns. Within the family, teenagers communicate more with family members through social media 
than directly interacting. Meanwhile, Barbato et al. [1] tested the relationship between family communication climate and interpersonal communication motives. Conversation Oriented families communicate with their children for relationship-oriented motives (affection, fun, relaxation), and conformity-oriented families communicate with their children for personal influence (control and escape) motives and for showing affection.

Children's personalities and adolescents are significant to be built through family communication patterns because family habits and informal education were very influential, especially for children and adolescents' personalities. Individuals from conversation-families showed greater self-disclosure levels, desire for control, self-esteem, and socializing. In contrast, those from conformity-oriented families were more likely to monitor themselves and were shy and restrained [13]. Model of implementation of evidence-based treatment in capacity building show in Figure 1.

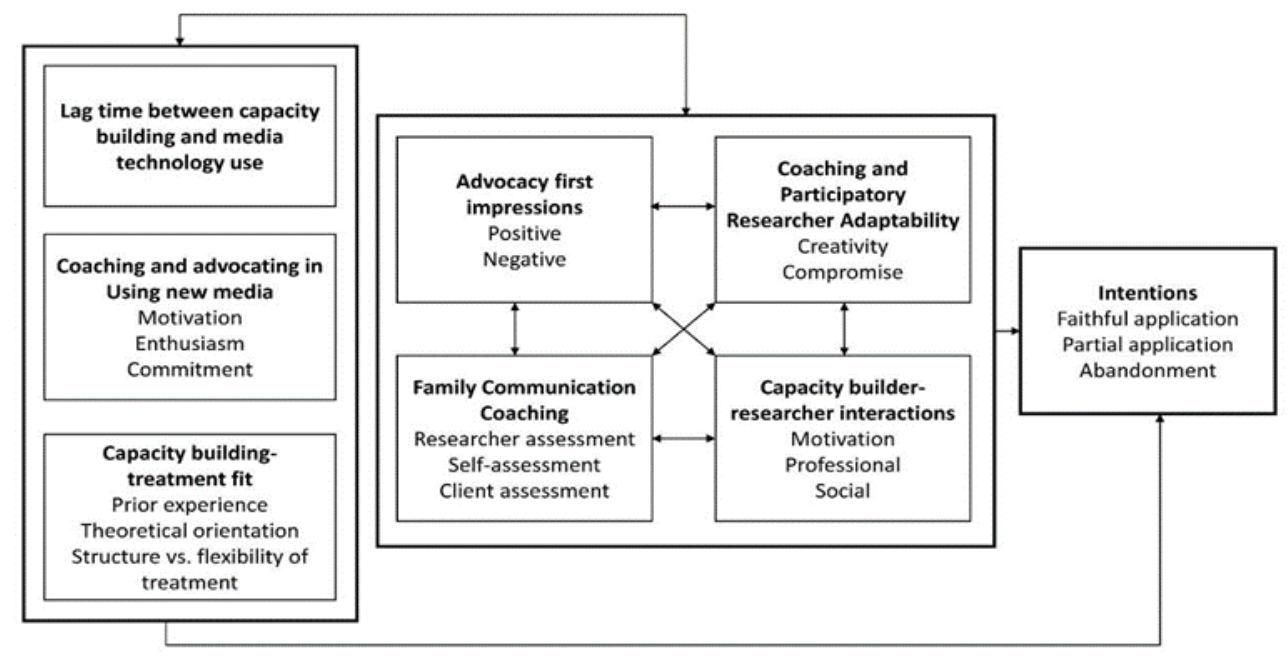

Fig. 1. Model of implementation of evidence-based treatment in capacity building

This research model discusses technology and society in a smaller scope on adolescents, families, and communities. We see the importance of a capacity building program, so that action research is an option besides ethnography with three months of observation, interviews, coaching, and accompanying.

This research article identifies current mental health in adolescents to carry out coaching, knowledge sharing, and mentoring to adolescent children. We also observed physical activity, health, and interviews to understand mental health. After the recovery program, we conducted observations and interviews again by observing the communication patterns of families and their relationships, whether there was a change for improvement or not.

\section{Methods}

Ethnography strengthened the method with three months long observation at the site (from January to March 2020). Researchers interact with adolescents, families, and the community 
while observing and interviewing. Ethnographic research such as O'Brien [24] on factors shaping indigenous mental health provides deeper insights into the notion of mental health. Capacity building with coaching and mentoring to every family in the community is the right way to improve adolescents' mental health. Action research and ethnography are the methods we choose. However, the criteria for families selected through the community of housewives called the Palmerah District PKK is the right sampling technique with 22 family groups. On average, each family has two children, so that the total sample is 88 people. Twenty-eight of them were interviewed with details of 14 children and 14 parents.

Regarding the researcher's intervention in the capacity-building program, this study's results are in line with Read et al. [27], who also intervened in rural Ghana's local communities. Communities living with mental illness, family members, and healers have a more significant potential to influence changes in the care of mental illness. Read also conducted research using ethnographic methodology as well as intervening.

Action research was carried out on communities in two villages involving 22 families as participants, and each family has one to four children. Ethnography was also used to collect data from observations, interviews, and researchers who are active in the community for three months. The first step is to identify the problem and then carry out an extension and mentoring program. Researchers applied a program to improve interaction and communication patterns in families, both dyadic and group. The empowering families and community are conducted through day-to-day coaching to the adolescents to improve communication patterns and relationships between family members, increasing knowledge about communication technology's function, and strengthening cultural ties face-to-face and online.

Ethnography focuses on accounting for the studied social agents' actions and intentions and outlining how such behavior is rationalized and understood by the wider group [21]. According to Lofland [22], the critical aspect of performing an ethnographic analysis is to balance the role of being an active part of the selected community while intermittently assessing emergent findings against the existing and developing theory. In effect, ethnography reveals the relationship between behavioral intent, attitudes, and beliefs against actual behavioral patterns [7].

Brenman et al. [3] also view the importance of intersecting cultures and mental health in the family, especially for children with disabilities. Therefore, his approach to ethnographic studies in children and adolescents' mental health services, the National Health Service in the United Kingdom, is based on transcultural psychiatry's theoretical perspective. Meanwhile, Snodgrass et al. [32] use a cognitive anthropological theory of "culture as socially transmitted knowledge." The ethnographic research approach was used to explain the socially learned game patterns in understanding online gaming experiences. Palinkas et al. [25] also conducted an ethnographic study of implementing evidence-based treatments in child mental health. Previously, Lee et al. [20] also used an ethnographic study of the experiences of depression among patients, as did Ware et al. [36], who used ethnographic methods with field observation, open-ended interviewing to investigate the meaning of mental health services. Thus, ethnography and capacity-building programs are the right methods to build families and communities in Palmerah County, West Jakarta.

Interactions and interviews were conducted with a set of semi-structured open questions related to online media use dimensions, including types and duration. The open-ended questions include mental health concepts, depression, physical health, dependence on online games, chatting, interaction with parents, and verbal and nonverbal communication with parents. The interview also focuses on the knowledge of children and adolescents about mental health and 
educational content, caring for people, parents' knowledge about good communication patterns, and the cultivation of good family values.

\section{Results and Discussion}

\subsection{The lag time between capacity building and media technology use}

Technology and society are two inseparable sides, but technology adoption always takes time for acceptance. Rapid change causes some communities or families to have no opportunity to adjust because technology suddenly must be faced and implemented. Experience and knowledge are still limited, causing some people not to place their role in media technology consumption. Children and adolescents accept the fact at their age that apart from having formal interactions at school, they are free to use various gadgets to interact online. Meanwhile, the average parent and school are inadequate in advocating their knowledge in online media consumption. No guidelines and signs support each family member in the social system as consumers and direct them to balance offline and online life. Some of the family, community, and social values built suddenly were dissolved by the revolution in communication technology. Children and adolescents who grew up with online digital products seemed to be separated from the older generation or their parents because they had no comparative practice before the advent of smartphones, tablets, social media, and online videogames. A housewife as participant-1 (48 years old) in the interview said:

In the early days of my Android smartphone's emergence, it was difficult to talk or invite children to gather in the family room because they were busy individually. They liked to get angry if I disturbed them because they did not respond when I called. Not only my children but also my husband, he was even more comfortable with his smartphone alone. This condition caused me to feel alone, and I became stressed until finally, I asked to buy a smartphone and stop subscribing to the landline. Yet, as a family, each of us had a smartphone and had new activities besides running the household. But we do not do many things like we used to. Never again gather in the family room, watch together, or go out to neighbors' houses. We have more time to interact on social media with our own family and neighbors. Life feels completely different than before.

Parents in many families in the community do not understand what is going on with a sudden significant change. Even though they can finally adjust, but with limited knowledge, they cannot provide experience and guidelines on how their children should use gadgets. The parents cannot coach their children in balancing the online and offline life so that they are not addicted to the internet, in which they spend all day except when sleeping. Thus, it will take time to increase the family's capacity to adopt reasonable media technology to avoid extreme dependence.

\subsection{Coaching and advocating in using new media}

\subsubsection{Motivation}

Researchers' involvement in families in the Palmerah County PKK (housewives) community aims to understand that teenagers and parents are accustomed to maintaining family communication patterns that balance online communication and direct interaction within the 
family and their peer groups. Researchers conducted coaching with the approach as a teacher who was actively involved daily for three months of ethnography. Coaching and advocacy in using new media are the researcher's activities in capacity-building programs. Researchers motivate children and adolescents to be enthusiastic about using the internet as a learning tool such as completing homework, question-and-answer and discussion with friends, and parental guidance channels. One teenager (15 years old) from participant-2's family said:

I do not want to be disturbed by my pleasure in playing with gadgets. But I also often feel sleepy in class. I feel sluggish and not enthusiastic about studying. Well, I will follow the advice to limit playing online games. I need help learning to do homework. I want my grades in school to be better than now.

\subsubsection{Enthusiasm}

Some parents pay attention to the decline in their children's school achievement, while others do not think about the decrease in their children's school achievement. Some families do not know if their children are experiencing stress due to school pressure and online game addiction. Some parents who have tertiary education feel concerned about the dependence of online games on their children. Parents like that have enthusiasm when researchers provide solutions for coaching and mentoring to their children. Although it may be that their appreciation for the lecturer or researcher has influenced community acceptance, the researchers' intervention was carried out professionally to act as a capacity-builder. Participant-16, a mother (42 years old), said:

We hope for help to understand my teenagers so that their high school scores will be good, and they can enter college. At first, I wanted to hire an informal tutor to make the exam work, but I am very grateful for the mentors' assistance from the advocacy team. We are very hopeful and very enthusiastic.

\subsubsection{Commitment}

The research team wants each family to commit to change the wrong or unbalanced communication pattern between direct interactions that reflect the care and cohesiveness of family members and online media use. However, for three months, the capacity-building program remembered that the PKK community had a dependency on the program's sustainability. A mother, participant-9 (37 years old), said that due to her busy schedule taking care of her business at Palmerah Market, she did not have time to supervise her children's activities when she came home from school and only met her child in her room before her child fell asleep. But even at night, her children often still play online games. She said, "I haven't been able to commit to change my son's habit of playing online games because he is with his friends, while his father and I are busy in the market".

\subsection{Capacity-building treatments appropriateness}

The housewife community or PKK community in Palmerah County, West Jakarta, is a capacity-building activity that involves a research team and family members in the city. The relationship between the research team and the community is very close, with researchers as coaches and mentors for children and parent groups. Capacity building treatments include experience, theoretical orientation, and structure versus treatment flexibility. 
Previous experiences for some families were very worrying because their children were addicted to online games and chatting or watching YouTube for excessive time. Although parents limit the amount of spending for online connections, their children in the area quickly get free wi-fi from many of their neighbors, such as apartments, inns, and residences that have open connections. Parents who are aware of the psychological and physical effects of excessive online media use on adolescent children experience worries.

The researchers involved in the community as educators have concerns for theoretical orientation. This role means the researcher pays attention to concepts, theoretical perspectives to be applied to the family regarding family communication, mental health endeavors, and efforts to balance online gadget usage with direct communication interactions with other family members and peer groups in the community. Researchers' roles as coaches and mentors for children are improving their study performance and having a healthier family relationship. Researchers also pay attention to the structures in the nuclear family, extended family, peer groups, communities, and the wider community influenced by conventional media coverage and various viral social media issues. Therefore, as coaches and mentors, researchers are flexible in their roles.

\subsection{Advocacy first impressions}

Many families do not think that researchers' role is to continue to pay attention to and assist their teenagers daily. The duration of mentoring in a day is around 2 to 3 hours in some families, taking turns for three months. The first impressions of the parents are reflected in the statement of participant-10 (a mother, 39 years old):

I am happy to be accompanied so that my child is not too addicted to online games. I thought the teacher who attended them only came once and just chili ago but was continually directing them to be better and pay more attention to their homework. I was surprised by the coaching of the researchers.

Some other parents were less appreciative of researchers who wanted to help their children reduce stress and mental health problems. Still, researchers tried to convince parents, mostly because the community understood the higher education institution's seriousness as coaches' affiliation.

\subsection{Coaching and participatory researcher adaptability}

Coaches with permission and parents provide direction and guidance to children and adolescents about accessing educational internet content. Researchers also provide knowledge and insight into the appropriate duration of internet access for mental and physical health. Parents provide support and direction to their children, and the researcher begins to provide psychological treatment and physical exercises (healing) to restore children's fitness. Creativity in physical play is needed to get children to do it independently when left by coaches. Some of the neighboring children were gathered to allow them to play in a peer group-however, there were still teenagers' refusal to be guided by coaches and mentors. When they were accessing the internet, for example, they tried to hide. Coaches always were friendly to approach them to reduce the duration of playing online games. However, in the end, the coaches succeeded in building relationships with children and adolescents. 


\subsection{Family communication coaching}

Family communication is a solution that is practiced in capacity building to heal children and adolescents who experience severe internet addiction. Researchers carry out the identification and assessment of children in each family and provide treatment such as healing and discuss solutions with parents. At first, many parents do not realize that their children were stressed and mentally unhealthy. Likewise, unhealthy physical conditions are due to lack of rest and high dependence, especially on online games. One mother, as participant-8 (35 years old), said:

I came to understand that mental health is related to excessive use of online gadgets. Indeed, if I pay attention, my child looks tired because he plays too many online games. I am grateful to be given explanations and insights from a team of lecturers from the university.

The sharing of knowledge between the researchers and parents in several families allowed them to carry out self-assessments. Most families could carry out self-assessments after being given insight into the importance of mental health and children's physical health related to internet media consumption. For researchers, this family care is a form of professional service for client assessments to obtain positive results and good progress for the community's mental health by empowering the Palmerah District PKK community.

\subsection{Capacity-building interactions}

In the interaction, the research team, as a capacity builder, tries to motivate each family in the community. However, because most of the community's children suffer from internet addiction, researchers must involve parents to build a new spirit in their families to interact with love and concern for one another.

Observation and interview data show that adolescents spend much time using gadgets, less direct interaction with their parents and families. Adolescents show that their attention is focused on the gadget screens, such as online games, YouTube, chatting, and children having difficulty interacting with their parents. Parents who are aware of this state complain, as participant-3 (a 37 years-old mother), puts it:

I often scold my teenager who spend all her time playing online games, day, and night, lacking sleep, and her eyes are always tired. My child is sick, and her learning achievement has declined.

The parents' confession occurred after they were given insight and awareness in the capacity building program process. During the three ethnographic months, the researcher recorded the findings and testimonies of the parents. Observations and interviews also found that adolescents experienced physical health problems. A mother as participant-7 (44 years old) said:

I saw the wrinkled face of my teenager. His eyes always looked sleepy and not cheerful, and he often had headaches. I sometimes massage his hands and fingers because he feels sore. I know that he takes too long-playing online games and chatting or watching YouTube.

Thus, adolescent respondents whose online activity duration was extended face unhealthy physical and mental health problems.

\subsection{Intentions}

The intention dimension is essential to be analyzed from the results of observations and interviews during ethnographic mentoring. Every family was expected to do a precise or faithful application or partial application to share knowledge and experiences. After the condition got 
better, the companion team left the community with a note that the organization still hopes for the capacity-building program's sustainability. On the other hand, there is no community abandonment on the capacity-building program during the mentoring process.

The results showed that loosened dependence on online games and the social media chats' duration caused mental health recovery. The community shows the improvement of interaction and relationship patterns in family communication. Meanwhile, dimensions such as knowledge about the role of communication technology and cultural ties in the community are considered necessary by the community to improve adolescents' mental health. Researchers do capacity building by empowering family communication, sharing knowledge with adolescents, and creating time limits for online and offline activities that involve families.

Mental health recovery is the primary goal of action research, where researchers are directly involved in advocacy, coaching, and mentoring adolescents in 22 family groups in the Palmerah County PKK community. At the end of the program, almost all family groups saw the benefits of this capacity building program and wanted continuous monitoring. A mother (45 years old) with two kids and a teenager as participant-15 says:

I am happy that my teenager is now healthier physically and mentally after the lecturers coached and mentored our family. We are aware of the importance of a healthy way of life. It turns out that the gadget must be used wisely. Thank you.

Mental health and physical health were recovered, and a balanced communication pattern between online and offline occurred after the researchers had provided advocacy for three months. During the capacity-building program, a friendly relationship occurs between the research team and families participating in the community. Changes for the better in family communication allow parents to be more concerned with their children's mental health and physical health.

\subsection{Discussion}

Results of this research confirmed some previous research, such as Lam's about the relationship between parental mental health, particularly depression, and their children's internet addiction status. Depression is a symptom of mental unhealthiness in children and adolescents. At that young age, children and adolescents cannot manage themselves unless their parents intervene in good family management.

Online communication is essential for children and adolescents' educational period, but it must be balanced with offline family communication patterns. Direct interaction, both dyadic and group communication when eating together or doing joint activities inside and outside the home, is essential. Verbal and nonverbal communication are needed, such as physical touch and parents' advice for children before adulthood. Verbal and nonverbal communication in the family occurs in interactions and conversations at the dining table, in the family room, in the bedroom, lawn, or when gardening. According to Koerner et al. [19], this conversation fosters understanding of family communication in maintaining family functioning.

Improving the family communication pattern in this research is a practical solution based on identifying problems in the field and healing treatment as the initial treatment for depressed and mentally unhealthy children, making this article distinctive. In the context of family communication, this study's results are in line with the findings of Schrodt et al. [30], who analyzed family communication patterns and their associations with information processing, behavioral, and psychosocial outcomes. They show that family communication patterns have a meaningful relationship with various cognitive activities, relational behaviors, and individual well-being. 
The capacity-building carried out in this research reflects the spirit of building a future for young people with more severe challenges. Now and the future challenges become complicated, especially those related to the development of the technological environment in business, organizations, society, and all aspects of life, as Santo et al. [29] mention, engaging urban youth through technology. This research article's findings are also in line with the findings of Varjas et al. [34] regarding four culturally specific themes identified, such as adult behavior and practices, youth perspectives on the present and aspirations for the future, and social factors. The code categories for developing culture-specific interventions are attitudes, beliefs, customs, expectations, norms, and cultural values; and reflect the ecological framework that guides research.

Furthermore, Schrodt et al. [31] highlighted the role of parental confirmation and affection as mediators of family communication patterns and children's mental well-being. It was revealed that confirmation and parental affection partly mediated the effects of conversational orientation and fully mediated the effects of conformity orientation on young adults and children's selfesteem and perceived stress. In more detail, for example, as a comparison, Garcia and Saewyc [8] found three thematic patterns identified as "mentally healthy," "mentally unhealthy," and "health promotion." Piotrowska et al. [26] also suggest things that are in line with this research's results that there is a need for father-inclusive and universal parenting intervention to reduce child conduct problems. Therefore, it is necessary to raise awareness of culture and immigration on adolescent mental health.

However, this study differs from Daley et al., who found that adolescents have a more holistic view of their health care. This research found that adolescents did not care about their health except after participating in a family capacity-building program to strengthen family understanding and discipline. However, the research of Yap et al. [37] and Cardamone-Breen et al. [4] recommend using online parenting guidelines to prevent adolescent depression and anxiety. Meanwhile, this research article's results put forward the balance between online and offline parental guidelines and even improve the overall family communication pattern.

The new finding as the novelty of this study's results is that action research positively impacts mental health recovery for a community. The capacity-building program is a direct practice solution as an intervention carried out by researchers to obtain results on proving previous assumptions. Identifying problems found in mental unhealthiness and depression among children and adolescents was derived from internet consumption and excessive duration. More than that, it turns out that parents pay less attention to the importance of good family communication to strengthen their affection. However, continuous assistance is needed to ensure that a family communication pattern supporting health, both physically and mentally, is maintained.

\section{Conclusion}

The capacity building program in Palmerah Country shows mental health recovery in SDGs by empowering family communication. Children and adolescents in most families in the community experience depression, decreased learning spirit, mental and physical fatigue due to the long duration of playing online games. Internet addiction occurs mainly because of easy internet access via wi-fi in community areas where many restaurants, small hotels, and residences are available. Children are free to get an internet connection. 
Mental problems occur in the family community in Palmerah District before the capacity building program. Children and adolescents had mental fatigue, physical sicknesses such as eye fatigue, pain in the fingers, head, neck, and body due to the body position when doing online interaction and activities such as playing the game. Parents do not realize their children are mentally unhealthy, except for physical unhealthiness due to internet addiction and a lack of communication patterns that support their physical and psychological health.

Capacity building through improved family communication patterns has changed adolescents' bad habits by reducing online interaction and playing games and implementing balanced physical and online interactions with parents and fellow peer groups in the community. Treatment with knowledge sharing, coaching, and mentoring is carried out to improve the frequency and duration of online gadget usage by children, adolescents, and parents. Assistance was carried out for three months to obtain recovery through balanced communication between online and offline.

Mental health recovery requires ongoing accompaniment and coaching to families and communities in Palmerah County to maintain mental health, physical health, family health, and strengthen each family's relationship in the community. Coaching and mentoring are family assistance in a capacity-building program in a relatively short time and have proven beneficial for the community. However, the implication is that similar programs must be carried out continuously, considering that the environment inside and outside the community consider good habits bothersome.

These action research findings' theoretical implications suggest multi-discipline future research to study family harmony and technology-friendliness in daily life. The sudden adoption of technology can cause a gap between cultural values in the family and community, which results in users' mental health disruption, especially children and adolescents who are in a period of psychological and physical growth and during formal education with a heavy academic burden. Action research that advocates for families and communities on an ongoing basis is needed for each generation.

\section{Acknowledgment}

This research was carried out with community development academic (CDA) grant support for the internal program of Binus University in 2020. For this reason, we thank all for the cooperation and will continue the capacity building program in the same location or other places as a form of community service.

\section{References}

[1] Barbato, C. A., Graham, E. E., \& Perse, E. M. (2003). Communicating in the family: An examination of the relationship of family communication climate and interpersonal communication motives. Journal of Family Communication, 3(3), 123-148.

[2] Bell, V. (2007). Online information, extreme communities and internet therapy: Is the internet good for our mental health? Journal of mental health, 16(4), 445-457.

[3] Brenman, N. F., Hiddinga, A., \& Wright, B. (2017). Intersecting cultures in deaf mental health: An ethnographic study of NHS professionals diagnosing autism in D/deaf children. Culture, Medicine, and Psychiatry, 41(3), 431-452. 
[4] Cardamone-Breen, M. C., Jorm, A. F., Lawrence, K. A., Rapee, R. M., Mackinnon, A. J., \& Yap, M. B. H. (2018). A single-session, web-based parenting intervention to prevent adolescent depression and anxiety disorders: Randomized controlled trial. Journal of medical Internet research, 20(4), e148.

[5] Connell, J., \& Dworkin, J. (2012). College students' information and communications technology (ICT) use with parents. Journal of the Association of Higher Education Parent/Family Program Professionals, 2, 2-17.

[6] Eade, D. (1997). Capacity-building: An approach to people-centred development. Oxfam.

[7] Eyles, J. (1988). Interpreting the geographical world: Qualitative approaches in geographical research. In J. Eyles \& D. Smith (Eds.), Qualitative methods in human geography (pp. 1-16). Totawa, NJ: Barnes \& Noble.

[8] Garcia, C. M., \& Saewyc, E. M. (2007). Perceptions of mental health among recently immigrated Mexican adolescents. Issues in Mental Health Nursing, 28(1), 37-54.

[9] Fitzpatrick, M. A. (2004). Family communication patterns theory: Observations on its development and application. Journal of Family communication, 4(3-4), 167-179.

[10] Floersch, J., Longhofer, J., \& Suskewicz, J. (2014). The use of ethnography in social work research. Qualitative Social Work, 13(1): 3-7.

[11] Galderisi, S., Heinz, A., Kastrup, M., Beezhold, J., \& Sartorius, N. (2015). Toward a new definition of mental health. World Psychiatry, 14(2), 231.

[12] Holmes, D. (2009). Computer-Mediated Communication, in Encyclopedia of Communication Theory, Littlejohn, S. W. \& Foss, K. A. (eds.), Thousand Oaks, CA: Sage.

[13] Huang, L. N. (1999). Family communication patterns and personality characteristics. Communication Quarterly, 47(2), 230-243.

[14] Jensen, K. B., \& Craig, R. T. (2016). The International Encyclopedia of Communication Theory and Philosophy, 4 Volume Set. John Wiley \& Sons.

[15] Keyes C. L. M. (2006). Mental health in adolescence: is America's youth flourishing? Am J Orthopsychiatry, 76: 395-402.

[16] Keyes C. L. M. (2014). Mental health as a complete state: how the salutogenic perspective completes the picture. In: Bauer GF, Hammig $€ \mathrm{O}$ (eds). Bridging occupational, organizational, and public health. Dordrecht: Springer: 179-92.

[17] Kirmayer, L. J., Raikhel, E., \& Rahimi, S. (2013). Cultures of the Internet: Identity, community and mental health.

[18] Koerner, A. F. (2009). Family Communication Theory. In Encyclopedia of Communication Theory, Littlejohn, S. W. \& Foss, K. A. (eds.), Thousand Oaks, CA: Sage, 381-385.

[19] Koerner, F. A., \& Mary Anne, F. (2002). Understanding family communication patterns and family functioning: The roles of conversation orientation and conformity orientation. Annals of the International Communication Association, 26(1), 36-65.

[20] Lee, D. T., Kleinman, J., \& Kleinman, A. (2007). Rethinking depression: an ethnographic study of the experiences of depression among Chinese. Harvard Review of Psychiatry, 15(1), 1-8.

[21] Ley, D. (1988). Interpretive social research in the inner city. In J. Eyles (Ed.), Research in human geography (pp. 121-138). Oxford: Blackwell.

[22] Lofland, J. (1976). Doing social life: The qualitative study of human interaction in natural settings. New York: Wiley.

[23] Morley, D., \& Parker, C. S. (2014). Understanding computers: Today and tomorrow, comprehensive. Cengage Learning.

[24] O'Brien, A. (2005). Factors shaping indigenous mental health: an ethnographic account of growing up Koori from a Gubba perspective. The Australian journal of holistic nursing, 12(1), 11-20.

[25] Palinkas, L. A., Schoenwald, S. K., Hoagwood, K., Landsverk, J., Chorpita, B. F., \& Weisz, J. R. (2008). An ethnographic study of implementation of evidence-based treatments in child mental health: First steps. Psychiatric services, 59(7), 738-746.

[26] Piotrowska, P. J., Tully, L. A., Collins, D. A., Sawrikar, V., Hawes, D., Kimonis, E. R., ... \& Dadds, M. R. (2020). ParentWorks: Evaluation of an online, father-inclusive, universal parenting 
intervention to reduce child conduct problems. Child Psychiatry \& Human Development, 51(4), 503513.

[27] Read, U. M., Adiibokah, E., \& Nyame, S. (2009). Local suffering and the global discourse of mental health and human rights: An ethnographic study of responses to mental illness in rural Ghana. Globalization and Health, 5(1), 13.

[28] Rudi, J. H., Walkner, A., \& Dworkin, J. (2015). Adolescent-parent communication in a digital world: Differences by family communication patterns. Youth \& Society, 47(6), 811-828.

[29] Santo, C. A., Ferguson, N., \& Trippel, A. (2010). Engaging urban youth through technology: The youth neighborhood mapping initiative. Journal of Planning Education and Research, 30(1), 52-65.

[30] Schrodt, P., Witt, P. L., \& Messersmith, A. S. (2008). A meta-analytical review of family communication patterns and their associations with information processing, behavioral, and psychosocial outcomes. Communication monographs, 75(3), 248-269.

[31] Schrodt, P., Ledbetter, A. M., \& Ohrt, J. K. (2007). Parental confirmation and affection as mediators of family communication patterns and children's mental well-being. The Journal of Family Communication, 7(1), 23-46.

[32] Snodgrass, J. G., Batchelder, G., Eisenhauer, S., Howard, L., Dengah, H. F., Thompson, R. S., ... \& Powell, C. (2017). A guild culture of 'casual raiding'enhances its members' online gaming experiences: A cognitive anthropological and ethnographic approach to World of Warcraft. New media \& society, 19(12), 1927-1944.

[33] Tracy, K. (2009). Community, in Encyclopedia of Communication Theory, Littlejohn, S. W. \& Foss, K. A. (eds.). Thousand Oaks, CA; Sage: 143-146.

[34] Varjas, K., Nastasi, B. K., Moore, R. B., \& Jayasena, A. (2005). Using ethnographic methods for development of culture-specific interventions. Journal of School Psychology, 43(3), 241-258.

[35] World Health Organization (2004). Promoting mental health: concepts, emerging evidence, practice (Summary Report). Geneva: World Health Organization.

[36] Ware, N. C., Tugenberg, T., Dickey, B., \& McHorney, C. A. (1999). An ethnographic study of the meaning of continuity of care in mental health services. Psychiatric Services, 50(3), 395-400.

[37] Yap, M. B., Martin, P. D., \& Jorm, A. F. (2018). Online parenting guidelines to prevent adolescent depression and anxiety: evaluating user characteristics and usefulness. Early intervention in psychiatry, 12(5), 951-958.

[38] Young, K. S., \& De Abreu, C. N. (Eds.). (2017). Internet addiction in children and adolescents: risk factors, assessment, and treatment. Springer Publishing Company. 